\title{
La religion, la loi et l'État dans I'Europe contemporaine : perspective comparative des principaux dilemmes
}

\author{
Ronan McCrea*
}

Résumé : Cet article traite des principaux éléments de la réglementation des liens entre la religion, le droit et l'État en Europe. Il analyse les principaux défis auxquels ces réglementations sont confrontées, et notamment la façon dont l'immigration et la diversité religieuse posent problème aux modèles existants. Enfin, l'article esquisse une comparaison entre les approches européennes et américaines, afin d'évaluer si les États-Unis peuvent constituer une source d'inspiration aussi bien pour les États que pour les institutions pan européennes.

Cet article traite de la façon dont est gérée la religion dans les sphères juridique et constitutionnelle. Il s'agit aussi d'évaluer dans quelle mesure ces sphères doivent s'adapter aux mutations de l'environnement religieux européen, tout en comparant les évolutions européennes avec celles des États-Unis. Certes, les États-Unis et l'Union Européenne sont des régimes politiques (polities) aux équilibres fort différents. Les États-Unis ont un sens beaucoup plus clair de leur identité légale, politique et culturelle, de sorte qu'il leur ait plus aisé d'imposer un cadre plus exigeant pour réguler les liens entre religion, droit et État sur leur territoire. En dépit de cette différence, il y a des similitudes significatives entre les deux rives de l'Atlantique. Les États-Unis et l'Union Européenne constituent tous deux des ensembles démocratiques, libéraux et de type fédéral où les autorités centrales doivent s'ajuster aux spécificités des États fédérés, dans le cas américain, ou des États membres, en Europe. Les deux ensembles sont caractérisés par un engagement fort en faveur de valeurs clairement revendiquées. Les États-Unis se targuent d'être le pays de la liberté et leur discours national est tout entier orienté vers la célébration de leurs valeurs fondatrices. L'Union Européenne, depuis plus de trente ans, a développé, avec ses traités fondateurs, une identité politique fondée sur la démocratie et les droits de l'homme. En même temps, une différence substantielle demeure entre les États fédérés américains et les États membres de l'UE. L'Alabama diffère de New York au moins autant que la Grèce et les Pays-Bas. Les Cours de

Senior Lecturer, Faculté de Droit, University College, Londres, et Jean Monnet Fellow au Centre Robert Schuman de I'Institut Universitaire de Florence (Italie). 
Justice et les Assemblées, aux États-Unis comme en Europe, font face à un défi identique, celui de résoudre l'engagement en faveur de leurs valeurs fondatrices tout en préservant l'autonomie morale de leurs unités constituantes. C'est sur la question religieuse que cette tension est particulièrement apparente.

Je commencerai par identifier les trois piliers qui définissent les approches de l'Europe à l'égard de la relation entre la religion, la loi et l'État, avant de voir comment la diversité religieuse croissante pose de nouveaux défis et quels changements devraient - ou non - être apportés aux configurations traditionnelles. Dans le même temps, je considèrerai la manière dont les ÉtatsUnis font face à de tels enjeux.

\section{Caractéristiques communes des approches européennes à l'égard de la religion, de la loi et de l'État : les trois piliers}

Il existe une divergence significative dans les approches qu'ont les différents États européens quant à la relation entre la religion, la loi et l'État. Certains ont des Églises officielles, certains reconnaissent plusieurs fois alors que d'autres sont officiellement laïques. Cependant, malgré la diversité qui existe en termes symboliques et institutionnels, il y a un chevauchement significatif entre les États. On peut clairement identifier un modèle européen qui est renforcé par les institutions paneuropéennes.

\section{Pilier 1 : Une vision individualiste de la religion}

À plusieurs reprises, la Cour européenne des droits de l'homme a insisté sur le fait qu'au regard de la Charte des droits fondamentaux la religion est « avant tout une question de liberté et de conscience individuelles $»^{2}$. Cet aspect est souligné dans l'Article 9 de la Convention Européenne des Droits de l'Homme $(\mathrm{CEDH})$ qui protège la liberté « de pensée, de conscience et de religion » et est illustré dans la jurisprudence de la Cour de Strasbourg qui a affirmé que le but principal de l'Article 9 était de protéger le droit des individus à choisir leurs croyances. L'autonomie institutionnelle et les aspects collectifs de la religion peuvent également être protégés, mais la Cour les voit comme des éléments secondaires dont la protection est souvent liée au besoin de garantir la liberté religieuse individuelle. Cette vision n'est pas universellement partagée. Pour beaucoup, les aspects communautaires et ritualistes de la religion sont de première importance. Alors que de nombreux États musulmans considèrent la prévention de l'apostasie de l'islam comme plus importante que les choix religieux individuels, beaucoup de pays en Europe perçoivent l'harmonie

2 Eweida and others v. UK (nos. 48 420/10, 59 842/10, 51 671/10 et 36 516/10). Jugement du 15 janvier 2013, paragraphe 80. 
religieuse comme une valeur plus importante que le choix individuel et la liberté d'expression. Il existe également des divergences entre les approches européennes et celle des États-Unis où le Premier Amendement ne s'intéresse qu'à la liberté de religion et ne mentionne pas celle de pensée ou de conscience et où les Cours ont adopté une vision plus absolutiste de l'autonomie religieuse institutionnelle. Par exemple, avec l'approche américaine, l'État est incompétent pour intervenir dans la relation entre un employé et un employeur religieux même si les responsabilités pastorales ne constituent qu'une toute petite partie des activités de l'employé. En revanche, la Cour Européenne des Droits de l'Homme cherche à équilibrer les droits des uns et des autres lorsqu'ils sont en conflit et impose aux États de définir entre employeurs religieux et employés une harmonie qui prenne en compte la proximité du travail en question avec la «mission proclamatrice» de la religion ${ }^{3}$.

\section{Pilier II : la séparation entre religion, loi et politique}

Bien que la religion et l'État soient souvent entrelacés, la séparation entre la religion d'un côté et la vie politique de l'autre constitue un pilier fondamental des approches européennes à l'égard de la religion dans la vie publique. Comme Mark Lilla le note, la séparation entre religion et politique n'est ni naturelle ni inévitable; c'est en fait un exercice intellectuel plutôt curieux qui résulte de circonstances propres à l'histoire européenne ${ }^{4}$. En outre, comme

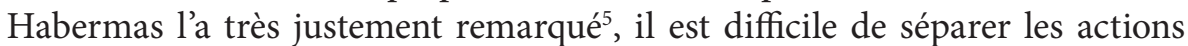
politiques des actions religieuses, particulièrement chez les croyants dont la foi impose une activité missionnaire et pour lesquels le salut éternel doit passer avant le respect des choix individuels en matière de foi et avant la préservation d'une harmonie œcuménique. L'exercice intellectuel qui consiste à séparer les croyances religieuses de la politique est néanmoins fortement établi en Europe. Les enquêtes Eurobaromètre indiquent que la religion a une très faible influence sur les convictions politiques individuelles ${ }^{6}$. La séparation de l'univers politique et de l'univers religieux est si établie en Europe qu'il s'agit souvent davantage d'une attente sociopolitique que d'un simple principe strictement juridique. Bien qu'une stricte séparation institutionnelle entre

3 Il y a ici un contraste flagrant entre l'approche de la Cour de Strasbourg dans Obst v. Germany (App. 425/03, jugement du 23 septembre 2010) et Schüth v. Germany (App. 1620/03, jugement du 23 septembre 2010) et celle de la Cour suprême des États-Unis dans Hosanna Tabor Evangelical Lutheran Church v. Equal Employment Opportunity Commission et al., 565 US_ (2012), qui fut unanimement décidée par les neuf juges.

4 M. Lilla, The Stillborn God, New York, Knopf Books, 2007.

5 J. Habermas, "Intolerance and Discrimination», vol.1, n 1, 2003, International Journal of Constitutional Law, p.2-7.

6 Eurobarometer 69, 2008. Disponible sur http://ec.europa.eu/public_opinion/archives/eb/eb69/ eb69_values_en.pdf (dernière visite : décembre 2013) 
l'État et la religion soit relativement rare en Europe, la législation des pays européens ne mentionne jamais de norme religieuse. En outre, la Cour Européenne des Droits de l'Homme a déclaré que toute théocratie violerait la Convention européenne des droits de l'homme; l'Union Européenne a elle clairement établi que le "sécularisme démocratique » était une condition d'adhésion? ${ }^{7}$ En revanche, les États-Unis ont une culture politique au sein de laquelle la religion joue un rôle bien plus important qu'en Europe, mais en même temps leur approche juridique est tout aussi restrictive à l'égard des tentatives d'utiliser la loi pour mettre en œuvre un enseignement religieux. La Cour suprême a non seulement affirmé que la Constitution rejetait les mises en application trop intrusives de la moralité sexuelle abrahamique traditionnelle, mais elle a aussi à plusieurs reprises déclaré que les lois s'appuyant seulement sur des justifications religieuses ne pouvaient pas être adoptées et que le corps législatif devait fournir des raisons séculières pertinentes pour les lois qu'il décidait d'approuver'.

\section{Pilier III : le chevauchement culturel}

Cette séparation entre religion et politique coexiste avec des liens institutionnels et culturels forts entre les États européens et certaines confessions religieuses. La plupart des États européens ont une foi majoritaire qui transparaît dans les arrangements communautaires et dans les symboles culturels nationaux. De nombreux drapeaux, comme ceux des pays scandinaves, portent une croix ; plusieurs jours de fête nationale, comme la Saint Patrick en Irlande, sont empreints de religion; dans des pays historiquement chrétiens, l'aménagement du temps de travail, avec les week-ends et les vacances, est structuré à la lumière des fêtes chrétiennes comme Noël ou Pâques. Des icônes culturelles nationales comme Notre-Dame-de-Paris, le livre de Kells (un évangile enluminé datant du IX ${ }^{\mathrm{e}}$ siècle) en Irlande ou la Pietà de MichelAnge en Italie sont également très liés à des traditions religieuses. C'est ainsi qu'un ordre politique séculier coexiste avec des symboles et des arrangements culturels et communautaires qui reflètent la prédominance historique et l'influence culturelle disproportionnée d'une foi particulière dans certains pays. Même dans le contexte américain, où les Cours ont cherché à limiter l'endossement symbolique d'une foi particulière de la part de l'État en déclarant comme anticonstitutionnels l'affichage des Dix Commandements, la prière à l'école publique et les crèches, il y a eu une reconnaissance limitée d'un nombre de pratiques religieuses culturellement établies lors de manifestations

7 Voir R. McCrea, Religion and the Public Order of the European Union, New York, Oxford University Press, 2014, chapitre 6.

8 Voir Lawrence v. Texas 539 US 558 (2003), Kiryas Joel Village School District v. Grumet 512 US 687 (1994), Stone v. Graham 449 US 39 (1980). 
publiques. Ainsi la devise «In God We Trust » sur les billets américains ou la phrase " May God Save This Honorable Court " prononcée au début des sessions de la Cour suprême ont été interprétées comme des exemples de " déisme cérémoniel " dont le rôle culturel et l'ancrage historique ont effacé toute signification religieuse spécifique ${ }^{9}$.

\section{Les défis posés aux modèles établis}

Le curieux mélange européen, fondé sur une approche individualisée à l'égard de la religion, une séparation de la religion et de la politique et des symboles culturels appartenant à une foi particulière, est de plus en plus mis à l'épreuve. La diversité religieuse grandissante soulève des problèmes complexes au sein de chaque " pilier ». Elle met particulièrement en exergue la tension qui existe entre une vision individualisée de la religion et une dimension religieuse intégrée à l'identité nationale collective. Une diversification des approches à l'égard de ce qui constitue les aspects essentiels de la religion est nécessaire au vu des défis qui attendent les modèles de protection de la liberté religieuse déjà établis. La présence grandissante de traditions religieuses qui distinguent moins la religion de la politique dans la sphère publique, engendre des conflits potentiels avec les normes politiques et juridiques existantes, ce qui nécessite de limiter l'influence religieuse dans le débat public. Enfin, l'affaiblissement du lien entre nationalité et foi individuelle pose de plus en plus la question de la place que l'État doit accorder aux symboles religieux : peuvent-ils représenter l'État?

\section{Dilemme I : La diversité religieuse et la liberté de religion}

La vision individualisée de la liberté de religion correspond surtout à une vision protestante et porte principalement sur la croyance et la conscience de l'individu. Laccroissement de la diversité religieuse en Europe et particulièrement la présence accrue de religions, comme l'islam, qui mettent moins l'accent sur la conscience individuelle de la religion que ne le fait le protestantisme occidental, ont renforcé des critiques de longue date contre la jurisprudence de la Cour Européenne des Droits de l'Homme à propos de l'Article 9. La Cour se voit reprocher de ne pas avoir assuré une protection suffisante aux aspects collectifs de la religion, notamment le rituel, ou à la religion comme mode de vie plutôt que comme système de croyance. Cumper remarque ainsi qu'elle a été accusée de ne pas soutenir les attentes des traditions

$9 \quad$ Lynch v. Donnelly 465 US 668 (1984). 
non-chrétiennes ${ }^{10}$. Evans a aussi remarqué que la Cour a rapidement reconnu un droit au prosélytisme, qui est au cœur de la tradition chrétienne, mais est beaucoup plus réticente devant la reconnaissance d'un droit à se vêtir en fonction de critères religieux, ce qui est secondaire dans le monde chrétien ${ }^{11}$.

Il est certainement important de faire en sorte que le manque de familiarité avec une foi ou un simple chauvinisme ethnique n'ait pas de conséquence sur le degré de liberté religieuse accordé aux groupes minoritaires par rapport aux religions culturellement établies. Cependant, la question ne doit pas être de savoir si la vision de la liberté religieuse qui sous-tend la jurisprudence de la Cour de Strasbourg est capable de couvrir tout ce qui peut être impliqué par le terme religion. La Convention Européenne des Droits de l'Homme est un document libéral qui défend la liberté religieuse et non pas la religion ellemême (quand elle a pris des décisions contre le discours anti religieux, elle l'a fait par souci de protéger les sentiments des croyants, mais pas pour protéger la religion) ${ }^{12}$. La liberté de pensée, de conscience et de religion est protégée, car elle fait partie d'un ensemble libéral qui protège le droit de l'individu de se construire une identité significative et appropriée ${ }^{13}$. Le travail de la Cour n'est pas d'identifier ce qui, d'un point de vue sociologique ou théologique, appartient à la religion, mais d'identifier ce qui va dans le sens de la liberté religieuse.

Une telle approche implique qu'une partie de ce qui est considéré comme religieux n'est pas forcément dans le cadre de l'Article 9. La loi est un outil imparfait auquel on demande de reconstruire des réalités sociales et émotionnelles complexes pour qu'elles puissent cadrer avec des principes généraux. Un tel processus ne pourra jamais capturer dans son entier la richesse des réalités qui seront traitées. La relation d'un employé et de son employeur implique souvent un engagement émotionnel important envers une carrière professionnelle qui constitue un aspect essentiel de l'identité de l'employé ; pourtant la loi ne peut aborder cet engagement que comme une forme d'échange, un travail contre une rémunération. Il en va de même pour la forme juridique du mariage

10 P. Cumper, "The Rights of Religious Minorities : The Legal Regulation of New Religious Movements " in P. Cumper, S. Wheatley, dir., Minority Rights in the 'New' Europe, La Haye, Martinus Nijhoff Publishers, 1999, p.174. La récente décision de la Cour, SAS v. France (juillet 2014, 43 835/11), qui accepte la loi française contre le port du « voile intégral » pourrait s'inscrire dans ce constat.

11 C. Evans, Freedom of Religion under the European Convention on Human Rights, Oxford, Oxford University Press, 2001, p.125. Notons cependant qu'Evans écrivait avant la décision Eweida v. United Kingdom (2010), qui a reconnu le droit d'un employé à porter une croix sur son lieu de travail.

12 Otto Preminger Institut v. Austria (1994), 19 EHRR 34.

13 Cette approche est aussi celle de I'Union Européenne : Guidelines on the Promotion and Protection of Religion or Belief, Conseil de l'Union Européenne, Réunion du Conseil des affaires étrangères, Luxembourg, 24 juin 2013. 
qui, contrairement au mariage religieux, ne représente que peu la réalité émotionnelle de la vie de couple. La loi ne reflète pas la pleine signification et la diversité de l'expérience religieuse. Comme dans d'autres domaines, elle se doit de réduire un phénomène social et émotionnel large et complexe à des éléments particulièrement précis et importants dont la protection doit être explicitée pour devenir un principe général.

Dans le contexte européen, la garantie de la liberté de religion opère aux côtés d'autres droits individuels à l'autonomie comme la liberté d'expression, le droit au respect de la vie privée, celui de ne pas être soumis à la torture et à la détention arbitraire, tandis que l'Article 9 couvre la liberté de pensée, de conscience et de religion. Tout ceci indique que l'essentiel de ce qui doit être protégé n'est autre que le droit individuel à l'autonomie en matière de croyance, un domaine où la religion est considérée comme fondamentale. Le sens de la communauté religieuse ou l'importance des rituels constituent pour beaucoup des éléments capitaux de leur foi, mais, pour la Cour, cela sera seulement le cas dans la mesure où ces éléments seront liés à la liberté de religion et centrés sur l'autonomie individuelle.

Cette approche de la protection de la liberté de religion a été critiquée comme ne protégeant pas assez les éléments collectifs de la religion ou des formes de religion qui ne sont pas aisément liées aux revendications d'autonomie individuelle ou associationnelle en matière de croyance.

Qu'une religion ne nous soit pas familière ou qu'elle soit exotique ne signifie pas que ses demandes de protection ne doivent pas être prises au sérieux. Comme le défend justement Laborde, il faut être vigilant afin que les structures existantes ne contiennent pas de préjugés injustifiables qui sont souvent un peu dissimulés ${ }^{14}$. Cependant, cela ne veut pas dire qu'un système qui protège la liberté de religion doive s'assurer que tout ce qui appartient à la religion soit traité comme ayant un besoin urgent de protection. La religion est une catégorie vaste et diverse qui couvre un large spectre de revendications et de comportements : certains sont très nobles et moralement convaincants, d'autres sont triviaux et futiles. Aucun système juridique ne peut ni ne doit espérer accorder une importance égale aux divers aspects des nombreux phénomènes qui constituent la religion. La question fondamentale est donc de savoir s'il y a de bonnes raisons pour considérer que les éléments religieux identifiés par la Cour européenne comme l'essence des droits protégés par l’Article 9 méritent davantage de protection. Jusqu'ici, la Cour a développé une jurisprudence selon laquelle la religion est un domaine ressortissant à la conscience et à l'autonomie de l'individu. Ce n'est pas simplement parce que la Cour aurait

14 C. Laborde, Critical Republicanism. The Hijab Controversy and Political Philosophy, New York, Oxford University Press, 2008. 
adopté une vision protestante de la religion, mais d'abord et avant tout parce qu'elle est la gardienne de l'interprétation d'une charte libérale de droits individuels. Elle conçoit donc, et ce à juste titre, la liberté de religion comme un élément d'un cadre libéral plus large visant à assurer l'autonomie individuelle sur des questions clés d'identité personnelle. Une charte libérale des droits fondamentaux conçoit le libéralisme religieux comme une des fondations de sa perception globale. Une telle perspective implique que les formes de religiosité qui sont plus difficiles à transcrire en termes de revendications favorables à l'autonomie privée recevront moins de protection. Ceci résulte inévitablement de l'engagement de la CEDH en faveur de la protection de valeurs libérales. Les États signataires se sont engagés à être des sociétés libérales qui protègent les principes libéraux.

La liberté religieuse collective et les droits communautaires ont souvent une relation très concurrentielle avec les droits individuels à l'autonomie (on peut citer comme exemple l'imposition d'un code moral comme condition nécessaire pour le recrutement d'employés dans les écoles et les hôpitaux religieux). En conséquence, il est parfaitement possible que les traditions religieuses mettant davantage l'accent sur les liens communautaires que sur le choix individuel en matière de foi rencontrent plus de difficultés à exprimer leurs revendications en termes plus susceptibles de correspondre à ceux d'une charte libérale des droits. Le droit de s'engager dans des croyances et des pratiques religieuses moins familières pour nous n'en est pas moins protégé par les textes européens. Cependant, il ne faut pas « jeter le bébé avec l'eau du bain » et saper l'orientation libérale et égalitaire de la liberté de conscience et de religion telle qu'elle a été construite par la loi européenne.

\section{Dilemme II : La religion en politique}

Le deuxième défi posé aux modèles déjà établis est lié au retour de la religion dans la sphère politique. Beaucoup de confessions bien établies culturellement, comme le catholicisme, n’ont pas complètement accepté les restrictions apportées à l'influence religieuse sur la loi et la politique. Ce ne fut qu'après le Concile de Vatican II que l’Église catholique adhéra explicitement à la liberté de religion et à la démocratie libérale. En outre, beaucoup de responsables religieux ont continué à jouer un rôle actif dans les débats sur les lois ayant trait à la question des droits des homosexuels, au mariage, à l'avortement et à l'euthanasie. En Europe cependant, il est devenu consensuel d'interdire aux autorités religieuses d'utiliser la loi pour protéger leurs valeurs et d'exercer une influence dominante sur la loi et la politique. Or ce n'est pas le cas dans les régions d'où sont originaires beaucoup de communautés de migrants en Europe. La religion joue un rôle politique décisif dans de nombreux pays, 
particulièrement dans le monde musulman et dans beaucoup d'États d'Afrique et du Moyen-Orient (à la fois chrétiens et musulmans). Dans de nombreuses régions du monde, c'est la loi qui est principalement utilisée pour mettre en vigueur des interdictions religieuses, par exemple contre l'homosexualité.

Olivier Roy a constaté qu'en Europe beaucoup se sont sentis menacés "par l'émergence de nouvelles communautés qui ne se sentent pas liées par les vieux compromis douloureusement institués » entre le religieux et le séculier ${ }^{15}$. La réponse des États européens et des institutions face à la nouvelle importance de la religion est allée dans deux directions. D’un côté, les institutions politiques ont cherché à impliquer les communautés religieuses dans le processus politique grâce à des programmes structurés de dialogue, comme celui mis en place par l'Union Européenne avec le Traité de Lisbonne ${ }^{16}$ (les États membres ont aussi pris des initiatives similaires). De l'autre, les États se sont montrés de plus en plus disposés à accepter des limites à l'influence de la religion sur la loi et aux principes religieux socialement controversés - comme l'égalité des sexes et l'homosexualité - pour en faire même des prérequis fondamentaux de tout ordre démocratique. Ainsi, lors du processus d’adhésion de la Roumanie à l'Union Européenne, il fut demandé aux autorités roumaines de résister à la pression de l'Église orthodoxe et de décriminaliser l'homosexualité, afin que le pays puisse devenir membre de l'Union. L'Union a en effet défini la «laïcité démocratique " (democratic secularism) comme condition sine qua non pour les pays souhaitant devenir membres. Cette évolution rend le modèle européen plus proche des États-Unis. Le modèle constitutionnel américain se base en effet sur une séparation rigoureuse de la religion et de l'État et insiste sur des raisons séculières suffisantes pour justifier une législation. Actuellement, l'Europe est aussi en train de faire du rejet de la théocratie et de tout exercice religieux du pouvoir de l'État une de ses normes constitutionnelles.

On retrouve cette convergence entre l'Europe et les États-Unis au niveau de leur approche face aux immigrants. Aux États-Unis, l'obtention dela citoyenneté américaine dépend depuis longtemps de la connaissance de la Constitution américaine et de ses normes constitutionnelles comme la démocratie et la liberté d'expression. En Europe, ces dernières années ont vu l'adoption d'une forme de «patriotisme constitutionnel » dans les procédures d'obtention de la citoyenneté. Les lois des États européens en la matière imposent depuis quelques années des tests d'intégration afin que les candidats indiquent qu'ils ont conscience - ou parfois même qu'ils déclarent accepter - des principes comme l'égalité des sexes et les droits des homosexuels s'ils veulent être naturalisés. En

15 O. Roy, Secularism Confronts Islam (traduction de George Holoch), New York, Columbia University Press, 2007, p.6.

16 Voir le Traité sur le fonctionnement de l'Union Européenne, Article 17. Disponible à : http://eurlex.europa.eu/legal-content/FR/TXT/?uri=CELEX:12012E/TXT (consulté en juin 2014). 
France, les autorités ont refusé d'accorder la citoyenneté à une femme à cause de sa « pratique radicale» de la religion et de son rejet de l'égalité des sexes ${ }^{17}$. Un homme s'est lui aussi vu refuser la nationalité française, car il n'acceptait pas que sa femme prenne la parole ou sorte de chez eux sans sa permission. Dans les deux cas, le refus fut justifié par le refus de ces citoyens français potentiels d'accepter les valeurs fondamentales de la République française ${ }^{18}$. Alors que les tests américains évitent explicitement les questions religieuses (le degré de différence entre la composition religieuse des populations migrantes et les autochtones étant bien plus faible aux États-Unis qu'en Europe), la conscience (et parfois l'acceptation) de certaines normes politiques et constitutionnelles est devenue un point fondamental du processus de naturalisation des deux côtés de l'Atlantique.

Dans certains pays (mais pas en France), les tests de citoyenneté ont été mis en œuvre de façon extrêmement discriminante. Beaucoup, à l'instar du Front National en France, se sont découvert un amour de la laïcité à partir du moment où ils ont pu l'utiliser comme un moyen pour combattre l'immigration. Dans certains États, les migrants occidentaux sont exemptés des tests de citoyenneté, mais les migrants venant d'États à majorité musulmane se doivent de faire preuve de leur bonne foi séculière lors d'un examen bien plus approfondi. Si une telle discrimination est inacceptable, ces tests peuvent néanmoins, s'ils sont pratiqués équitablement, servir des objectifs importants. La théocratie et le recours à la loi pour imposer des valeurs religieuses, comme la persécution des homosexuels en Ouganda et en Iran ou l'oppression des femmes en Arabie Saoudite, sont incompatibles avec la démocratie libérale. Les lois relatives à l'immigration autorisent souvent certains États à privilégier les migrants qui ont des liens ethniques ou linguistiques avec leur pays de destination ou ceux qui sont instruits, qualifiés, jeunes ou en bonne santé, en bref ceux qui présentent des caractéristiques attirantes. Il ne paraît pas non plus déraisonnable de privilégier parmi des migrants potentiels - qui deviendront peut-être des citoyens - ceux qui croient en la tolérance et en l'égalité au détriment de ceux qui ne peuvent se résoudre à accepter l'égalité des sexes, l'État laïque ou l'homosexualité.

17 Conseil d'État, Section du contentieux, $2^{\text {e }}$ et $7^{e}$ sous-sections réunies, Séance du 26 mai 2008 — Lecture du 27 juin 2008, n² 286798 - Mme M. Disponible à : http://www.conseil-etat.fr/fr/selection-de-decisions-du-conseil-d-etat/analyse-n286798-mme-m.html (consulté en juin 2014).

18 Voir "Algerian-born man living in France is refused French nationality », The Guardian, 9 juin 2011, http://www.theguardian.com/world/2011/jun/09/man-refused-french-nationality (consulté en mars 2014). 


\section{Dilemme III : Diversité et culture}

Avec l'accroissement de la diversité religieuse, le dernier défi est celui de la résonance religieuse des symboles culturels nationaux résultant d'un parcours confessionnel propre.

Comme le paysage confessionnel des États européens s'est diversifié, la capacité d'une seule foi particulière à représenter toute une identité nationale commune à tous a diminué. C'est en partie parce qu'il y a beaucoup de communautés ethniques qui ne partagent pas les allégeances culturelles chrétiennes ; c'est aussi parce que le nombre d'athées et d'agnostiques autoproclamés est en constante augmentation. Le recensement de 2011 au Royaume-Uni montre une augmentation de 15 à $25 \%$ du nombre de gens déclarant ne pas avoir de religion ${ }^{19}$. Auparavant, beaucoup de ceux qui nétaient pas particulièrement religieux se contentaient de se décrire comme culturellement chrétiens. En Europe, le nombre de ces chrétiens de nom a longtemps été supérieur à ceux qui se revendiquaient comme profondément attachés aux principes de la foi chrétienne. Mais au fur et à mesure que la religion et l'identité nationale se sont séparées, l'identité religieuse est devenue davantage une question d'idéologie et de croyance que le résultat de l'appartenance à une communauté nationale. Ceux qui ne sont pas de véritables croyants nont plus hésité à passer d'une identité chrétienne de nom à une identité plus clairement non-religieuse.

Une fois que la nationalité n'est plus synonyme d'une confession religieuse particulière, les symboles de cette religion perdent leur capacité à être des emblèmes culturels nationaux consensuels. Alors que ces symboles étaient autrefois partagés et reconnus par tous, ils sont maintenant extrêmement contestés. En Irlande, en 2007, un sikh, Ravinder Singh Oberoi, contesta les règles de l'uniforme de la Garda Reserve, la force volontaire de la police irlandaise, en demandant l'autorisation de porter le turban ${ }^{20}$. À Saint-Marin, en 1999, trois nouveaux membres du Parlement allèrent jusqu'à la Cour européenne pour contester le traditionnel serment qu'ils devaient faire ; ils dénonçaient la référence aux «saints évangiles » qu'il contenait et considéraient qu'elle violait leurs droits en tant que députés non chrétiens ${ }^{21}$. En Italie, en 2011, Soile Lautsi, une mère de famille athée, attaqua l'école de ses enfants devant la Cour Européenne des Droits de l'Homme car elle dénonçait la présence d'un crucifix dans les salles de classe. Au Royaume-Uni, la National Secular Society

19 Voir R.McCrea, "Is Migration Making Europe More Secular? » Aeon Magazine, juin 2013 : http://aeon.co/magazine/world-views/ronan-mccrea-secular-europe/ (consulté en mars 2014).

20 R. McCrea, Ibid.

21 Buscarini v. San Marino (1999) 30 EHRR 208. 
a intenté une action en justice pour contester les prières avant les réunions municipales et dans les écoles publiques.

Pourtant ces actions contre le symbolisme religieux public n'ont pas toutes été couronnées de succès. La police irlandaise a déclaré qu'elle se devait de rester religieusement neutre (bien que leur insigne soit basé sur une imagerie issue de l'art monastique celte et chrétien, qui est un aspect important de l'héritage national culturel irlandais) et la Haute Cour d'Irlande rejeta la revendication de $\mathrm{M}$. Oberoi ${ }^{22}$. La victoire initiale de $\mathrm{M}^{\mathrm{me}}$ Lautsi à Strasbourg fut renversée en appel ; les Juges expliquèrent que le «symbole passif » que constitue une croix sur un mur de classe n'était pas suffisamment endoctrinant pour nécessiter l'intervention de la Cour européenne ${ }^{23}$. Quant à la National Secular Society et sa dénonciation des prières, il se pourrait que sa victoire devant les tribunaux ${ }^{24}$ soit suivie par une défaite politique, car le gouvernement britannique a promis de légiférer pour renverser cette décision et les discussions sont en cours. Néanmoins, la prolifération des contestations contre la présence, dans la vie publique, de ces symboles chrétiens résiduels montre à quel point, dans une Europe plus diverse, l'héritage chrétien au sein des institutions publiques est de plus en plus controversé.

L'approche des Cours américaines sur le sujet a toujours été bien plus strictement séculière que dans la plupart des pays européens. Pour la loi européenne, les liens symboliques qui existent entre un État et une foi particulière ne deviennent problématiques que si leur proximité est telle qu'ils deviennent oppressifs de la liberté des autres (Lautsi v. Italy). Aux États-Unis, n'importe quel signe visible de foi religieuse est constitutionnellement problématique. Cela a créé une jurisprudence complexe et confuse ${ }^{25}$. La Cour suprême se contorsionne pour décider si, par exemple, la présence de symboles comme une menora (un chandelier juif à sept branches) ou un arbre de Noël pouvent rendre une crèche suffisamment séculière ${ }^{26}$. La différence d'approche entre les Cours américaines et européennes n'est pas difficile à expliquer. Même si les responsables politiques des deux ensembles sont ouvertement critiques de toute théocratie, il manque aux documents européens - les traités de l'Union Européenne et la Convention européenne des droits de l'homme un équivalent du Premier Amendement américain qui sépare explicitement l'État et la religion. De plus, les Cours européennes ne sont qu'une variation des Cours internationales, de sorte que leurs légitimités démocratiques sont

22 Oberoi and Another v. Commissioner of An Garda Síochána 2013 [IEHC] 267.

23 Lautsi v. Italy 30814/06 [2009] CEDH 1901 (3 novembre 2009).

24 National Secular Society \& Anor, R (sur l'application de) v. Bideford Town Council [2012] EWHC 175 (Admin) (10 février 2012).

25 Voir par exemple Van Orden v. Perry 545 US 677 (2005), McCreary County v. ACLU 545 US 844 (2005), Salazar v. Buono 559 US 700 (2010).

26 Ibid. (McCreary County v. ACLU). 
bien plus faibles que la Cour suprême américaine. La définition de normes rigoureuses dans un domaine si controversé est bien plus risquée pour les Cours de Strasbourg ou du Luxembourg parce quelles ont moins de moyens d'empêcher les États de contester leurs décisions.

En dépit de la réticence des Cours européennes à s'impliquer dans de tels problèmes, il n'y a pas d'échappatoire : plus les symboles et les arrangements nationaux ont un caractère religieux spécifique, plus il sera difficile d'intégrer les membres de minorités religieuses dans la société et de gagner leur allégeance à une citoyenneté nationale partagée. En outre, plus le sens d'une citoyenneté multiconfessionnelle est faible, plus il s'avère difficile d'obtenir des individus engagés religieusement l'acceptation de compromis au nom de la citoyenneté ou du besoin de vivre ensemble harmonieusement.

Néanmoins, comme le suggère Charles Taylor, les communautés politiques durables ne sont pas " une équipe constituée par un peu d'histoire commune partie de zéro, sans rien de plus à partager que les passagers d'un vol international $»^{27}$. Les « communautés imaginées " qui constituent les États nation ont besoin d'un certain degré d'identité communautaire et d'évènements partagés provenant d'une histoire singulière. Cette histoire singulière augmente l'importance des symboles de foi, des identités et des institutions qui l'ont influencée. Les circonstances historiques spécifiques de chaque histoire nationale s'accorderont mieux avecl'identité et les croyances de certains groupes par rapport à d'autres. En Grande-Bretagne, les partisans de la république par exemple vivent dans un État où la monarchie est une caractéristique centrale de l'histoire nationale, ce que Churchill appelait « la longue continuité de nos institutions ». Il est ainsi inévitable qu'en Grande-Bretagne beaucoup d'évènements communautaires nationaux, s'ils veulent avoir une quelconque résonance historique, aient avoir un lien avec la monarchie. Dans l'idéal, il serait sans doute positif que les célébrations nationales soient basées sur une tradition plus égalitaire, mais nous ne sommes pas dans l'année zéro et la culture ne peut pas être créée à partir de rien.

Les célébrations nationales ou collectives qui ne sont pas liées à une tradition plus ancienne ne parviennent généralement pas à capturer l'imaginaire de l'opinion. Par exemple, la promotion de la Journée de l'Europe par l'Union Européenne n'entraîne aucun véritable sentiment d'appartenance collective. Ainsi en Grande-Bretagne, la seule alternative aux évènements collectifs liés à la royauté serait qu'il n'y ait plus d'évènement du tout ; mais par la même occasion, il y aurait moins de possibilités de créer une allégeance commune à

27 C. Taylor, «Liberal Politics and the Public Sphere » Discussion Paper 15 (The Centre for the Study of Global Governance, London School of Economics, 1995), p.19. 
tous les segments de la société britannique. Le résultat serait une société plus atomisée et solitaire qu'elle ne l'est déjà.

Dans beaucoup de pays, les évènements nationaux ont une dimension religieuse toute particulière. En Irlande, la Saint Patrick est devenue une célébration de l'identité et de la culture nationales et un moyen pour les citoyens de se sentir reliés par leur engagement partagé pour leur société et sa tradition culturelle. L'histoire de la célébration elle-même est celle de l'arrivée du christianisme en Irlande, de sorte que la fréquentation des églises augmente ce jour-là. Dans l'ensemble, les non-Chrétiens oublient volontiers les échos religieux de la Saint-Patrick pour mieux adhérer à la célébration de l'appartenance nationale. Il y a néanmoins des limites au degré d'acceptation que l'on attend de la part d'une population religieusement diverse à l'égard des privilèges symboliques dont bénéficie la foi culturellement établie. Quand un symbole aux origines religieuses (comme le drapeau suédois) a développé une signification postreligieuse indépendante, il est raisonnable de s'attendre à ce que tout le monde accepte sa présence dans des lieux publics. On pourrait même défendre l'idée selon laquelle les symboles explicitement religieux comme la Pietà de MichelAnge peuvent être perçus pour leurs seules qualités artistiques; il serait alors raisonnable d'utiliser la Pietà comme un symbole culturel de l'État italien, comme un patrimoine culturel et artistique dont tous les Italiens, de toutes les confession - ou d'aucune - , seraient fiers.

Des symboles comme un crucifix sur le mur d'une école publique sont bien plus difficiles à appréhender en termes inclusifs et non confessionnels. Même si, dans le cas de Lautsi, la Cour Européenne n’a pas jugé le symbole assez oppressant pour intervenir, un crucifix ne peut bénéficier ni d'une signification post-religieuse ni d'une justification artistique pour justifier sa présence. Il peut donc de facto être perçu comme un moyen de préserver un degré de spécificité religieuse dans l'espace public, ce qui est inapproprié et inutile dans une société en pleine diversification.

Les cultures nationales ne sont pas intangibles. Leurs équilibres sont en négociation permanente et la loi européenne tente de conserver une attitude à la fois critique et ouverte dans le rendu de ses décisions. Les cultures nationales européennes sont confrontées à la nécessité d'intégrer des fois et des cultures dont la présence à grande échelle est un phénomène récent. L'enjeu est dès lors de changer pour refléter les apports et les craintes d'une population plus diverse. Une telle évolution ne doit pas pour autant impliquer une cassure totale avec le passé. Elle doit au contraire permettre aux cultures nationales de maintenir un sentiment de proximité avec leurs citoyens actuels ainsi qu'avec les générations passées et futures. 


\section{Conclusion}

Les approches européennes à l'égard de la relation entre la loi, la religion et l'État résultent d'une histoire très spécifique. Cette histoire fut marquée par l'important rôle historique du christianisme dans l'identité de la plupart des États européens et par la longue lutte entre les autorités religieuses chrétiennes et les forces séculières et humanistes (il se peut que le sécularisme soit né partiellement de la théologie chrétienne ; il s'est cependant avéré être un « enfant » rebelle qui s'est à plusieurs reprises opposé à son " parent » religieux au cours de l'histoire européenne). L'immigration, liée à d'autres facteurs, a entraîné une diversité religieuse de plus en plus importante en Europe. Avec une diversité de points de vue plus importante sur des sujets comme ce qu'implique le terme religion, ce que doit être l'objectif principal de celle-ci et le rôle approprié qu'elle doit jouer dans la vie juridique et politique, il n'est pas surprenant que les modèles déjà établis de gestion de la religion soient de plus en plus remis en question.

Face à la diversité grandissante, les États européens doivent éviter de s'attacher inconsidérément aux modèles établis. Il est bien trop commode de ne pas voir ou de ne pas prendre en compte le potentiel d'exclusion contenu dans les équilibres hérités du passé, qui ont été établis sans tenir compte des fidèles de confessions dont la présence à grande échelle en Europe remonte à quelques décennies à peine. Il convient d'avoir une attitude ouverte, critique et réflexive à l'égard des moyens qui sont déjà en place pour réguler la relation de la religion à la loi, à la société et à l'État.

Lorsqu'on évalue si les structures déjà existantes doivent être adaptées ou maintenues, la question clef n'est pas de savoir si ces structures sont ou ne sont pas compatibles avec les revendications qu'une foi en particulier peut formuler. Après tout, les demandes faites au nom de la religion sont nombreuses et variées. Certaines sont inoffensives, parfois même louables, d'autres le sont bien moins et sont même parfois potentiellement perturbatrices de l'ordre public, voire clairement menaçantes pour la liberté, le droit à l'intimité et l'égalité.

Les sociétés européennes devront réévaluer la relation entre la loi, la religion et l'État à la lumière d'une diversité religieuse croissante. Ce processus devra refléter les objectifs des structures établies. Les principes juridiques et les lois qui limitent l'influence de la religion dans la sphère politique permettent d'assurer la stabilité de sociétés qui sont en permanence divisées sur des questions de vérité religieuse et de protéger les intérêts de ceux qui, comme les femmes et les homosexuel (le) s, souffrent, dans beaucoup de régions du monde, de lois résultant d'interprétations patriarcales des religions. Le simple fait que certaines traditions religieuses ne soient pas habituées à se voir imposer des bornes ou rejettent les principes qui limitent, grâce à la loi, les valeurs 
religieuses, ne peut en aucun cas justifier l'abandon de normes au cœur de la construction européenne.

L'affaiblissement des liens traditionnels entre une foi particulière et l'identité nationale est néanmoins inévitable. La continuité d'une tradition culturelle spécifique et la préservation des liens avec un passé partagé et imaginé sont nécessaires, mais comme les sociétés européennes, longtemps mono-confessionnelles, ont dorénavant une plus grande diversité religieuse, cette dernière ne peut qu'être reconnue, avec comme conséquence directe un amoindrissement de la dimension religieuse des symboles culturels et nationaux. Si les drapeaux sont maintenant des symboles perçus indépendamment de leurs origines religieuses, ceux qui ont un caractère religieux plus explicite, comme les crucifix, posent encore problème. Plus les symboles nationaux ont un caractère religieux spécifique, plus il sera difficile de définir une identité nationale commune et non confessionnelle qui permettra de gagner la loyauté des minorités religieuses tout en préservant les liens avec un passé partagé et imaginé. Cette tâche est d'autant plus difficile en Europe où, contrairement aux États-Unis, l'immigration ne constitue pas le socle des histoires nationales.

Il est probable qu'au fur et à mesure que les catégories culturelles entourant la religion en Europe deviendront plus diverses, la régulation de la relation entre la religion, l'État et la loi ou par des normes culturelles tacites deviendra de plus en plus difficile. Au Royaume-Uni par exemple, l'accord implicite selon lequel les lois sur le blasphème ne seraient jamais ou très rarement utilisées pour réprimer les moqueries à l'égard de la religion n'a pas survécu à l'affaire des Versets sataniques et à la tentative des chrétiens évangéliques de censurer Jerry Springer : the Opera. Le délit de blasphème fut complètement aboli en 2008. Dans plusieurs États européens, les présupposés culturels exprimant une certaine réticence vis-à-vis de la religion dans les lieux publics ont aussi été remplacés par des normes juridiques explicites restreignant la présence de symboles religieux dans des environnements liés à l'État ou dans la vie sociale ${ }^{28}$.

Ce sera une tâche extrêmement difficile que de continuer à définir des normes juridiques explicites dans un tel domaine et la référence américaine sera de peu d'utilité. La liberté religieuse n'est pas du tout définie de la même façon dans les textes fondateurs que sont la Convention Européenne et la constitution américaine. Par ailleurs, les États-Unis sont une nation, et pas une union d'États souverains (même si les droits des États sont un thème essentiel de la vie politique américaine). La Cour Suprême est donc bien plus légitime dans son rôle de clarification des liens entre religion, droit et État, alors que les Cours européennes ont une autorité qui dépend largement de la coopération

28 Voir par exemple la loi française de 2004 sur les signes religieux dans les écoles publiques et la loi de 2010 sur l'interdiction du port du voile intégral dans les lieux publics en France et en Belgique. 
des États membres. De plus, la nature des changements auxquels les autorités européennes font face est différente de celle des États-Unis. Les États européens ont des liens avec des confessions précises bien plus forts que ce que les ÉtatsUnis n'ont jamais eu. La neutralité symbolique des signes religieux aux ÉtatsUnis, constamment rappelée par la Cour Suprême, est hors d'atteinte pour les Européens. Beaucoup de pays européens ont aussi une histoire d'affrontements religieux et de séparation antagoniste entre l'État et l'Église qui n’a jamais existé aux États-Unis. Enfin, le degré de différence religieuse entre les immigrants et les populations autochtons est bien plus faible aux États-Unis qu'en Europe. Autrement dit, les liens entre immigration et religion dans la sphère publique aux États-Unis ne sont pas aussi évidents qu'en Europe.

Cela étant, un certain degré de dialogue juridique transatlantique est évident sur certains points. En 2003, la décision de la Cour Suprême de décriminaliser l'homosexualité faisait référence à la décision sur le même sujet par la Cour Européenne des Droits de l'Homme en $1981^{29}$. De même, la décision de 2013 de la Cour Européenne des Droits de l'Homme - Eweida et al. v. United Kingdom - discutait la jurisprudence américaine et canadienne sur la liberté religieuse, sans pour autant reprendre une approche identique dans le texte final. L'Union Européenne et les États-Unis partageant la même tradition libérale occidentale, le dialogue entre les deux rives de l'Atlantique parait logique et les approches partagent d'ailleurs des similitudes. Néanmoins, les différences de cadre général, notamment le lien entre immigration et retour du religieux que l'on voit en Europe, signifient que le Vieux Continent doit définir son propre modèle. Un processus de clarification réfléchi devrait permettre de définir un équilibre entre d'un côté une approche critique des normes existantes et une ouverture vers le changement et de l'autre, une conscience des continuités historiques au sein des États Européens. Certains éléments de ce processus de renégociation demeurent non négociables. Le féminisme, le sécularisme, un certain lien avec un passé partagé et imaginé, ainsi qu’une éthique de la citoyenneté et un engagement à préserver la coexistence religieuse constituent autant d'éléments importants dans l'approche de nombreux États et ils ne seront pas abandonnés. Dire qu'une norme serait discriminatoire (en ce sens qu'elle serait trop difficile à respecter pour ceux qui appartiennent à une tradition religieuse particulière) ou qu'elle est une tradition nationale ne devrait pas suffire à condamner ou à maintenir la norme en question. Les règles que les traditions religieuses misogynes ou intolérantes ont des difficultés à accepter peuvent avoir un caractère d'exclusion ponctuelle, mais elles ont aussi de gros avantages en termes de liberté religieuse et de protection de la liberté de pensée. L'Histoire est jonchée de traditions qui ont été remises en question

29 Lawrence v. Texas, 539 US 558 (2003); Dudgeon v. UK (1981), ECHR 5. 
et justement abandonnées, car jugées inutiles et synonymes d'exclusion. En résumé, les responsables publics à qui incombe la délicate tâche de s'adapter à la diversité religieuse doivent garder à l'esprit que tout ce qui est existe déjà n'est pas forcément bon, mais qu’une partie de ce qui existe déjà est là pour de bonnes raisons.

Texte traduit de l'anglais (britannique) par Anne-Claire Lévy. $\nabla$

\section{Bibliographie indicative}

P. Cumper, S. Wheatley (dir.), Minority Rights in the 'New' Europe, The Hague, Martinus Nijhoff Publishers, 1999.

N. Doe, Law and Religion in Europe, New York, Oxford University Press, 2011.

C. Eisgruber, L. Sager, Religious Freedom and the Constitution, Cambridge MA, Harvard University Press, 2007.

C. Evans, Freedom of Religion under the European Convention on Human Rights, Oxford, Oxford University Press, 2001.

J. Klausen, The Islamic Challenge: Politics and Religion in Western Europe, New York, Oxford University Press, 2005.

C. Laborde, Critical Republicanism. The Hijab Controversy and Political Philosophy, New York, Oxford University Press, 2008.

M. Lilla, The Stillborn God, New York, Knopf Books, 2007.

S.Mancini, M. Rosenfeld, Constitutional Secularism in an Age of Religious Revival, New York, Oxford University Press 2014.

R.McCrea, Religion et Ordre juridique de I'Union européenne, Bruxelles, Bruylant-Larcier, 2013.

R. McCrea, Religion and the Public Order of the European Union, New York, Oxford University Press, $2^{\mathrm{e}}$ ed. 2014.

R.McCrea «Is Migration Making Europe More Secular? » Aeon Magazine, juin 2013.

S. Peers et al., The EU Charter of Fundamental Rights, Oxford, Hart Publishing, 2013.

G. Robbers (dir.), State and Church in the European Union, Baden-Baden, Nomos, 2005.

O. Roy, Secularism Confronts Islam (traduit par George Holoch), New York, Columbia University Press, 2007.

L. Tribe, American Constitutional Law, New York, Foundation Press, 2000.

R. White, C. Overy et al., The European Convention on Human Rights, New York, Oxford University Press, $5^{\mathrm{e}}$ ed. 2010. 